\title{
Compact, Thermally Tunable Silicon Racetrack Modulators Based on an Asymmetric Waveguide
}

\author{
Hui Yu, Marianna Pantouvaki, Member, IEEE, Sarvagya Dwivedi, Peter Verheyen, Guy Lepage, Roel \\ Baets, Fellow, IEEE, Wim Bogaerts, Member, IEEE, Philippe Absil, and Joris Van Campenhout, \\ Member, IEEE
}

\begin{abstract}
A compact wavelength tunable $10 \mathrm{~Gb} / \mathrm{s}$ silicon racetrack modulator with integrated thermo-optic heater is demonstrated using a waveguide with asymmetric cross section, combining the compact footprint of microdisk modulators with the design simplicity of regular racetrack or ring modulators. The outer perimeter of the asymmetric racetrack modulator is fully etched to maximize optical confinement, while the inner waveguide edge is shallowly etched to maintain an electrically conductive path to the embedded p-n diode and to control the propagation of the asymmetric optical mode and its coupling to the bus waveguide. Resistive heating elements based on highly doped Si strips are implemented at the outer edge of the modulator for thermo-optic control. The asymmetric modulators can be fabricated along with Si wire waveguides and shallowly etched fiber grating couplers using a simple process flow involving just two Si pattering steps. Devices with a bending radius of $10 \mu \mathrm{m}$ and a novel " $T$ "-shaped $p$-n diode layout have been fabricated and exhibit electro-optic modulation and heater efficiencies of $28 \mathrm{pm} / \mathrm{V}$ and $42 \mathrm{pm} / \mathrm{mW}$, respectively. At $10 \mathrm{~Gb} / \mathrm{s}$, a stable extinction ratio of $10 \mathrm{~dB}$ is demonstrated from a $2 V_{p p}$ drive swing, which can be maintained over a wavelength range of $\sim 4.6 \mathrm{~nm}$ by thermally tuning the modulator. This is equivalent with a temperature variation of about $62^{\circ} \mathrm{C}$.
\end{abstract}

Index Terms-Electrooptic modulators, integrated optics, optical resonators, thermooptical effects.

\section{INTRODUCTION}

$\mathrm{R}$ equirements for modulators in silicon photonic interconnect systems include CMOS compatibility, high extinction ratio ( $\sim 10 \mathrm{~dB})$, low insertion loss, high modulation speed (>10 Gb/s), low power consumption, and thermal robustness[1]-[3]. Carrier-depletion ring modulators potentially posses all these merits, provided that its temperature sensitivity is compensated, either actively by integrated heaters [4], [5] or passively by a modified Mach-Zehnder interferometer (MZI) [6]. Since the diode capacitance of a carrier-depletion ring modulator is proportional to its perimeter, its power consumption can be reduced by miniaturizing the ring. For example, a modulation energy and

Manuscript received October 5, 2012.

H. Yu, S. Dwivedi, R. Baets and W. Bogaerts are with the Photonics Research Group, Department of Information Technology, Ghent University-imec, Center of Nano- and Biophotonics, St. Pietersnieuwstraat 41, 9000 Gent, Belgium (phone:32-9264-3593; e-mail: hyu@ intec.ugent.be).

M. Pantouvaki, P. Verheyen, G. Lepage, P. Absil, and J. Van Campenhout are with imec, Kapeldreef 75, 3000 Leuven, Belgium. bit rate of $7 \mathrm{fJ} / \mathrm{bit}$ and $25 \mathrm{~Gb} / \mathrm{s}$ for a ring of $7.5 \mu \mathrm{m}$ radius [4], and $10 \mathrm{fJ} / \mathrm{bit}$ and $12.5 \mathrm{~Gb} / \mathrm{s}$ for a racetrack ring of $10.4 \mu \mathrm{m}$ radius [7] have been demonstrated. In order to realize a small radius without causing too much bending loss, deeply etched rib waveguides are necessary [4], [7]. On the other hand, several passive optical devices use fully etched Si waveguides, while others such as fiber grating couplers and splitters are preferably implemented with a shallow etch that produces a reduced lateral index contrast [8]. This means that typical silicon photonics circuits with modulators require 3 etch depths in silicon. However, for some real cases only two etch depths are implemented to cut the fabrication cost, although this compromises the performance of relevant elements. In [9] the grating couplers which are designed to use the same etch depth as the deep-etched ring modulator exhibit high coupling loss, and thus become a bottleneck of the whole photonic link. In contrast the ring modulators in [10] adopt the same etch depth as the shallow-etched grating coupler. The insufficient etch depth constrains the ring radius to $40 \mu \mathrm{m}$. An alternative to reduce the bending radius is to use a microdisk which only requires a complete Si etch [11]. The drawback is the presence of high order radial modes, and moreover, controlling the coupling strength and the resonance wavelength of a whispering-gallery mode is not as straightforward as the racetrack or ring resonator.

In this paper we demonstrate a compact racetrack modulator with an asymmetric waveguide core and a specifically designed doping pattern. Using only two etch steps necessarily shared with passive components, the particular waveguide geometry combines advantages of the ring and the microdisk. Its fully etched outer wall provides a tight confinement similar to a microdisk, while the shallowly etched inner edge enables us to engineer the coupling and the resonance like a ring, facilitating the implementation of a wavelength-division multiplexed (WDM) transmitter. This scheme also enables a doped silicon heater in close proximity to the ring. Stable operation is demonstrated at $10 \mathrm{~Gb} / \mathrm{s}$ over a wavelength range of $\sim 4.6 \mathrm{~nm}$ by thermally tuning the resonance wavelength.

\section{DESIGN AND FABRICATION}

The 3-D schematic diagram and the top view of our design are shown in Figs. 1 (a) and 1(b). The modulator is fabricated on a $200-\mathrm{mm}$ SOI wafer with $2 \mu \mathrm{m}$ buried oxide and $220 \mathrm{~nm}$ top c-Si layer. The outside of the ring is completely etched to 
expose the buried oxide layer, whereas the inside of the ring is shallowly etched by $70 \mathrm{~nm}$, i.e., the optimal etch depth for the fiber grating coupler. The asymmetric waveguide core width is $450 \mathrm{~nm}$. The bus waveguide has the same shape at the coupling section to maximize phase matching. The optical intensity profile of the fundamental mode is shown in Fig. 1(c).The effective index and the group index of this mode are 2.56 and 3.88 respectively, at $1550 \mathrm{~nm}$ wavelength. In order to achieve critical coupling, a racetrack structure is utilized with a bending radius of $10 \mu \mathrm{m}$ and a straight section length of $11 \mu \mathrm{m}$. The gap between the ring and the bus waveguide is $180 \mathrm{~nm}$. The asymmetric bus waveguide is tapered to $450 \mathrm{~nm}$ strip waveguides by two adiabatic tapers of $10 \mu \mathrm{m}$ each.

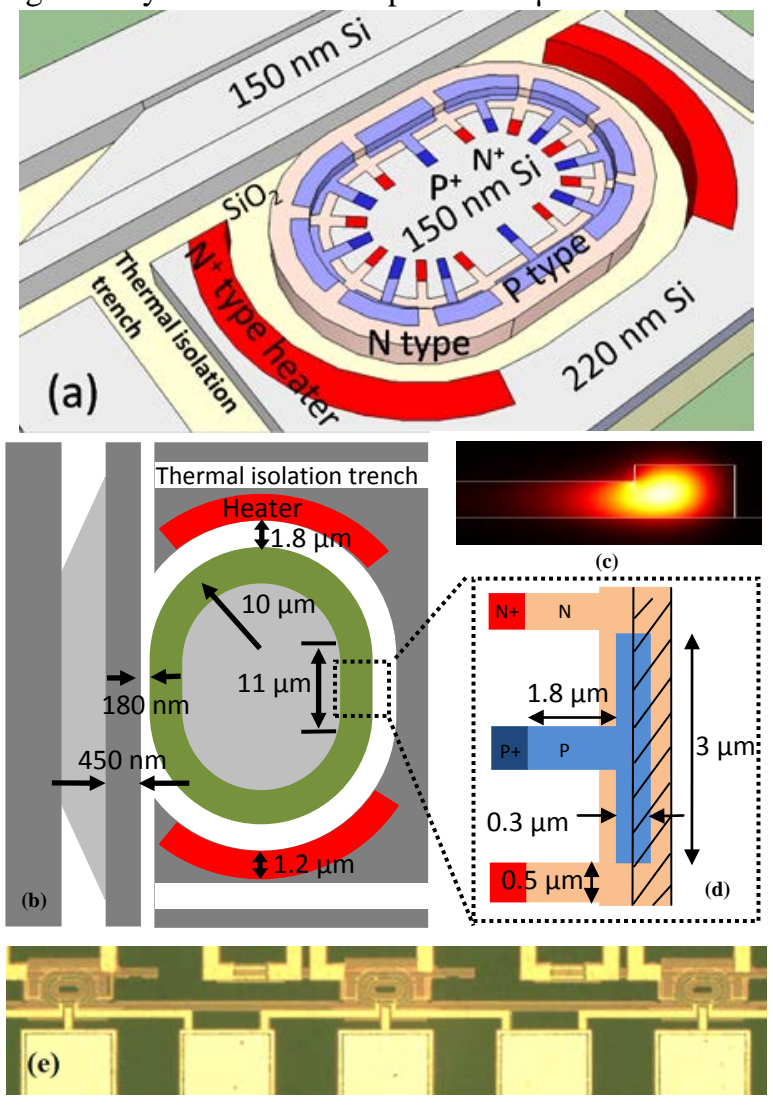

Fig. 1. (a) 3-D schematic diagram of the ring modulator based on the asymmetrical waveguide. (b) Top view of the ring modulator. (c) Optical intensity distribution of the fundament mode in the asymmetric waveguide. (d) Top view of the doping pattern where the shaded area delineates the position of waveguide. (e) Microscope image of a WDM transmitter based on 4 cascaded ring modulators. Only 3 of them are visible because of the limitation of the field of view.

Unlike the regular rib waveguide based modulator where $\mathrm{N}^{+}$ and $\mathrm{P}^{+}$contact regions are located at the opposite sides of the waveguide, the asymmetric waveguide design requires that both contact regions are situated on the inner side of the ring since the silicon outside the ring is completely etched. For this purpose, a particular doping pattern as shown in Fig. 1(d) is implemented within the asymmetric waveguide. The P-type silicon in has a "T" shape and is surrounded by an N-type area. Doped bridges offer electrical paths from the P-N junction to the contact areas inside the ring. Their width and height are $1.8 \mu \mathrm{m}$ and $0.5 \mu \mathrm{m}$ respectively. P-type strips in the N-type background have a width and a length of $0.3 \mu \mathrm{m}$ and $3 \mu \mathrm{m}$ respectively. The nominal doping concentration of the P-N junction is $1 \times 10^{18} / \mathrm{cm}^{3}$, whereas the contact areas are heavily doped to $1 \times 10^{20} / \mathrm{cm}^{3}$ to form an ohmic contact.

As there is no metal contact of high thermal conductivity outside the ring which dissipates the thermal flow, the heater can be implemented by the doped silicon in close proximity to the ring as shown in Figs. 1(a) and 1(b). Here, the heater was placed 1.8 um away from the ring. Compared to the metal heater on top of the ring [5], or the doped silicon heater embedded inside the ring waveguide which shortens the modulation section length [4], this scheme neither requires any additional metallization steps nor impairs the modulation efficiency. The device was fabricated in the $200 \mathrm{~mm}$ CMOS pilot line of imec. A full description of the processing flow can be found in [12].

\section{STATIC MEASUREMENT}
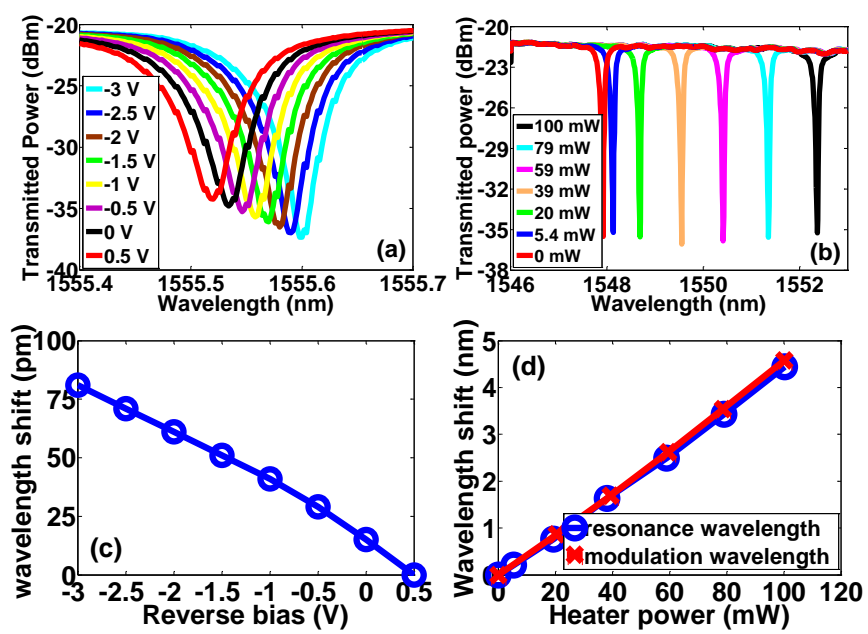

Fig. 2. (a) Transmission spectra of the ring for different reverse bias voltages. (b) Transmission spectra of the ring for different heater powers. (c) Resonance wavelength shift vs. the reverse bias. (d) Shifts of the resonance wavelength and the modulation wavelength vs. the heater power.

The measurement is at first carried out in the DC regime. The transmission spectra under different reverse bias voltages on the PN junction and different heater powers are shown in Figs. 2(a) and 2(b) respectively. The $Q$ factor and the free spectrum range (FSR) of the racetrack at $0 \mathrm{~V}$ bias are 13100 and $7.3 \mathrm{~nm}$ respectively. From these spectra we extract the resonance wavelength shift $\Delta \lambda$ vs. the reverse bias in Fig. 2(c) and vs. the heater power in Fig. 2(d). Provided a voltage swing of $1.5 \mathrm{~V}_{\mathrm{pp}}$ from $-1 \mathrm{~V}$ to $0.5 \mathrm{~V}$, the maximum DC extinction ratio is $11 \mathrm{~dB}$ at $1555.56 \mathrm{~nm}$ accompanying with an insertion loss of $4 \mathrm{~dB}$.

In Fig. 2(c), $\Delta \lambda$ for a voltage swing from $0.5 \mathrm{~V}$ to $-3 \mathrm{~V}$ is shown to be $81 \mathrm{pm}$. The modulation efficiency of our device compares favorably with the reported ring modulators based on deeply etched rib waveguides. The spectrum shifts for the same voltage swing are $77 \mathrm{pm}$ for a modulator with a doped silicon waveguide heater in [4], $58 \mathrm{pm}$ for a modulator with a Ti heater in [5], $80 \mathrm{pm}$ for a modulator with interleaved P-N junctions in [13], and $93 \mathrm{pm}$ for the modulator in [7]. The comparison indicates that the DC modulation efficiency of our scheme is not inferior to deeply etched rib waveguide based modulators.

The resistance of the heater is measured to be $1500 \mathrm{ohm}$ at 
room temperature. Its tuning efficiency is $45 \mathrm{pm} / \mathrm{mW}$ as shown in Fig. 2(d), which implies that $162 \mathrm{~mW}$ is required to shift the spectrum by a whole FSR. In contrast, the tuning power for one FSR is $66 \mathrm{~mW}$ for the ring with a doped waveguide heater in [4], and $46 \mathrm{~mW}$ for the ring with a Ti heater in [5]. The relatively poor efficiency originates from the low thermal conductivity of the $1.8 \mu \mathrm{m} \mathrm{SiO}{ }_{2}$ trench between the heater and the racetrack. We can improve the efficiency by reducing the width $w$ of the doped silicon heater and the gap $g$ between the heater and the ring, as well as by removing the undoped parts of the Si heater that act as an unwanted heat spreader. The horizontal temperature distributions along the waveguide center (the dashed line in the inset of Fig. 3) are shown in Fig. 3 for different values of $w$ and $g$ with a heater power of $50 \mathrm{~mW}$. The inset of Fig. 3 depicts the two-dimensional temperature distribution for $w=g=0.6 \mu \mathrm{m}$. Our present design uses a $1.2 \mu \mathrm{m}$ wide heater and a gap of $1.8 \mu \mathrm{m}$. As shown in Fig. 3, by shrinking the heater and the gap to $0.6 \mu \mathrm{m}$, the waveguide temperature rises by a factor of 2.2, which corresponds to a tuning power of $74 \mathrm{~mW}$ for one FSR. This is comparable with the heaters in [4], [5]. The optical intensity distribution in Fig. 1(c) indicates that a trench of $0.6 \mu \mathrm{m}$ still provides sufficient lateral confinement. On the other hand, as show in Figs. 1(a) and 1(b), the rectangular thermal isolation trenches are far from the heaters at the bottom of the arc, which impairs the effect of thermal isolation. As such, reshaping these thermal isolation trenches also helps to improve the heater efficiency. With all these approaches, we expect a heater power of $<50 \mathrm{~mW}$ to tune the wavelength by one FSR. Further improvements include the backside substrate removal technique which can reduce the heater power by more than tenfold [14].

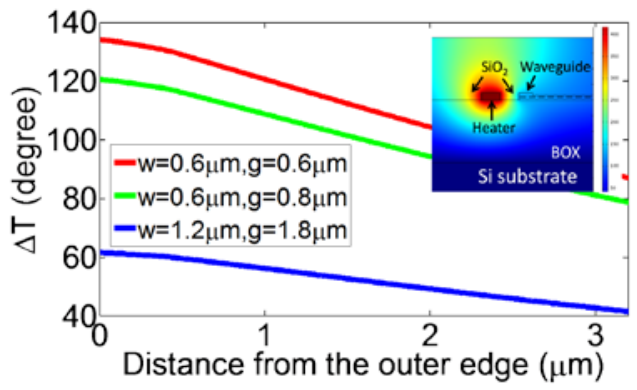

Fig. 3. Horizontal temperature distributions along the center of the asymmetrical waveguide with a heater power of $50 \mathrm{~mW}$. The inset shows a temperature profile for the waveguide cross section of $w=\mathrm{g}=0.6 \mu \mathrm{m}$.

\section{DYNAMIC MEASUREMENT}

The dynamic characterization includes the RF reflection coefficient measurement (the S11 parameter), the frequency response measurement of the electro-optical (EO) modulation (the S21 parameter), and the eye diagram measurement. The measured S11 is shown in Fig. 4 (a) from $100 \mathrm{MHz}$ to $40 \mathrm{GHz}$ at $0 \mathrm{~V}$ bias, where the inset is the circuit used to represent the modulator. $C_{0}$ is the capacitance of the GSG pads, $C_{1}$ and $R_{1}$ represent the capacitance and the series resistance of the diode. Since both $\mathrm{P}^{+}$and $\mathrm{N}^{+}$contact regions locate inside the ring, there are pin junctions between these contact regions. Two additional elements $C_{2}$ and $R_{2}$ are used to represent these pin junctions. We extract values of these elements from the S11 measurement result by curve fitting, which are: $C_{0}=30.6 \mathrm{fF}$, $C_{1}=75.8 \mathrm{fF}, C_{2}=19.8 \mathrm{fF}, R_{1}=606 \Omega$, and $R_{2}=83.3 \Omega$. Dashed lines in Fig. 4(a) depict the fitted curves, which agree well with the measured data in the whole frequency range. In principle, the bandwidth of a ring modulator is limited by both photon lifetime and the RC constant. The ring quality factor of 13100 here leads to a photon lifetime limited 3-dB bandwidth of 14.8 GHz. It is much higher than the bandwidth of the circuit in Fig. 4(a). As such, the frequency response of the circuit dominates the EO modulation bandwidth. With the circuit, the 3-dB bandwidths of $|\mathrm{S} 21|$ and $|\mathrm{S} 21|^{2}$, i.e., the optical and the electrical $3 \mathrm{~dB}$ bandwidths of the EO modulation, are calculated to be $6 \mathrm{GHz}$ and $3.5 \mathrm{GHz}$ respectively. The measured frequency responses of $|\mathrm{S} 21|^{2}$ are shown in Fig. 4(b) from $100 \mathrm{MHz}$ to $20 \mathrm{GHz}$ for bias voltages of $0 \mathrm{~V}$ and $-2.5 \mathrm{~V}$. At $0 \mathrm{~V}$, the $3 \mathrm{~dB}$ bandwidths are measured to be $5.9 \mathrm{GHz}$ for $|\mathrm{S} 21|$ and $4.1 \mathrm{GHz}$ for $|\mathrm{S} 21|^{2}$, which generally agrees with the prediction using the S11 measurements and the circuit in Fig. 4(a). A reverse bias of $-2.5 \mathrm{~V}$ boosts the $3 \mathrm{~dB}$ bandwidths to 9.1 GHz for $|\mathrm{S} 21|$ and $5.7 \mathrm{GHz}$ for $|\mathrm{S} 21|^{2}$.
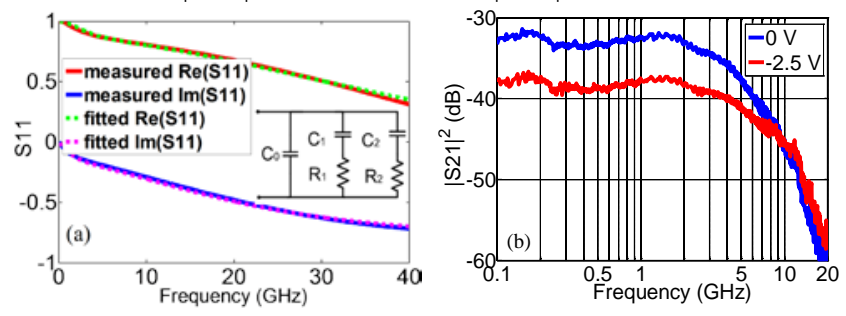

Fig. 4. (a) The $\mathrm{S} 11$ parameter of the ring modulator with $0 \mathrm{~V}$ bias. (b) The frequency response of the EO modulation.

The small-signal measurements show that the modulation bandwidth is mainly limited by the high series resistance of the diode. As shown in Fig. 1(d), the P-N junction is connected to the contact regions by bridges of $1.8 \mu \mathrm{m} \times 0.5 \mu \mathrm{m}$. A majority of the total resistance comes from these narrow paths. This can be relieved by expanding the bridge width and cutting the distance from the PN junction to the heavily doped $\mathrm{P}^{+}$and $\mathrm{N}^{+}$regions. On the other hand, the PIN diodes between the $\mathrm{P}^{+}$and the $\mathrm{N}^{+}$ contact regions can be removed by completely etching the silicon in between. In addition, our simulation confirms a bending radius of $5 \mu \mathrm{m}$ with negligible bending loss, which implies the $\mathrm{PN}$ junction capacitor $C_{1}$ can also be reduced. With all these measures, it is estimated that the final $3 \mathrm{~dB}$ bandwidth of $|\mathrm{S} 21|^{2}$ can exceed $11 \mathrm{GHz}$.

Eye diagrams are recorded under different heater powers by sending a $10 \mathrm{~Gb} / \mathrm{s}$ non-return-to-zero (NRZ) pseudorandom binary sequence (PRBS) to the device through a high speed GSG probes. The peak-to-peak voltage, pattern length, and bias voltage of the source PRBS signal are $2 \mathrm{~V}_{\mathrm{pp}}, 2^{31}-1$ and $-2.5 \mathrm{~V}$ respectively. Due to the superposition between the input and the reflected RF signals, the practical voltage swing on the diode is around $4 \mathrm{~V}_{\mathrm{pp}}$. Taking the large diode series resistance into account, the final voltage swing on the diode capacitor which is responsible for the modulation is around $2.6 \mathrm{~V}$. To measure eye diagrams, we could either adapt the heater power to the assigned operation lengths, or adapt the operation length 
to the assigned heater powers. In other to compare the shifts of the optimal modulation wavelength and the resonance wavelength, we use the same heater powers as shown in Fig. 2(b), and then tune the wavelength to achieve the best performance. The corresponding eye diagrams are presented in Fig. 5, where a heater power of $100 \mathrm{~mW}$ tunes the operation wavelength by $4.581 \mathrm{~nm}$ (63\% FSR). This indicates an average temperature rise of $62.6{ }^{\circ} \mathrm{C}$ in the ring according to $\Delta T=\Delta \lambda \cdot \lambda /\left(F S R \cdot L_{\mathrm{r}} \frac{\partial n_{\text {eff }}}{\partial T}\right) \cdot$ Here $n_{\text {eff }}$ is the effective index of the waveguide. As the heater power goes up from $0 \mathrm{~mW}$ to $100 \mathrm{~mW}$, the extinction ratio remains $10 \mathrm{~dB}$, while the quality of the eye falls only slightly from 7.37 to 6.77 (Qeye $>6$ corresponds to a bit error rate of less than $10^{-9}$ ). The dynamic ER is reduced to $8 \mathrm{~dB}$ if a signal of $1 \mathrm{~V}_{\mathrm{pp}}$ is used to drive the modulator. We expect a bit rate of $>20 \mathrm{~Gb} / \mathrm{s}$ after increasing the modulation bandwidth with the aforementioned measures.

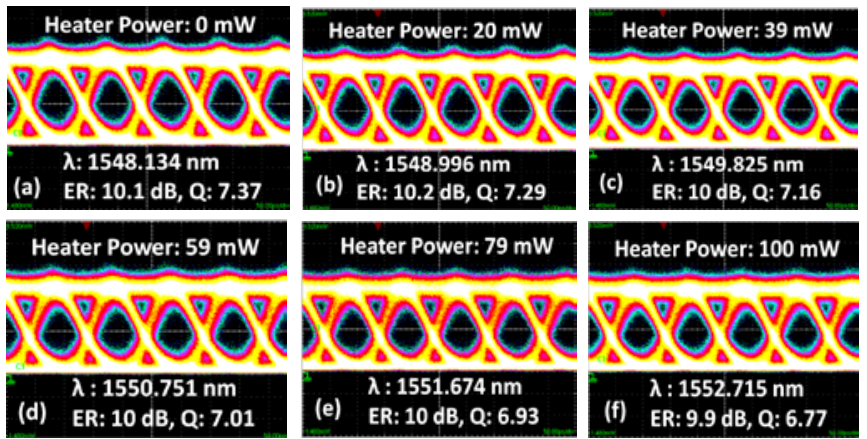

Fig. 5. Eye diagrams at different operation wavelengths and heater powers.

\section{WAVELENGTH-DiViSiOn MULIPLEXED TRANSMitTER}

The spectrum of a WDM transmitter which consists of 4 cascaded ring modulators is shown in Fig. 6, whereas its microscope image is shown in Fig. 1(e). A circumference difference of $0.12 \mu \mathrm{m}$ between two adjoining rings is implemented for a targeted channel spacing of $1.6 \mathrm{~nm}$ (200 $\mathrm{GHz}$ ). The deviation of the resonance wavelengths from their ideal spectral positions in Fig. 6 due to the fabrication non-uniformity can be compensated by the integrated heaters.

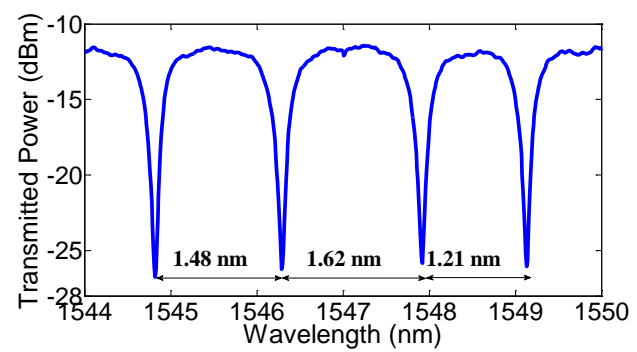

Fig. 6. Spectrum of a WDM transmitter consisting of 4 ring modulators.

\section{CONCLUSIONS}

We demonstrate an asymmetric racetrack modulator combining the compact footprint of microdisk modulators with the design simplicity of regular racetrack or ring modulators. The device can be fabricated along with optimized passive devices without introducing any additional Si patterning steps and enables straightforward implementation of integrated heaters based on doped Si strips. When driven by a $10 \mathrm{~Gb} / \mathrm{s}$ PRBS signal with $2 \mathrm{~V}_{\mathrm{pp}}$ swing, a fabricated device with a $10 \mu \mathrm{m}$ radius exhibits a stable ER of $10 \mathrm{~dB}$ while a heater power of $100 \mathrm{~mW}$ tunes its operation wavelength over a range of $4.581 \mathrm{~nm}$. By simply optimizing relevant design parameters, we expect that an improved device can finally operate beyond $20 \mathrm{~Gb} / \mathrm{s}$ with a driving voltage of less than $1 \mathrm{~V}_{\mathrm{pp}}$. In addition, the heater power to tune the operation wavelength by one FSR can be reduced to less than $50 \mathrm{~mW}$.

\section{ACKNOWLEDGMENT}

The authors acknowledge the efforts of imec's 200-mm pline for their contributions to the device fabrication. This work was supported by imec’s Core Partner Program.

\section{REFERENCES}

[1] A. V. Krishnamoorthy, R. Ho, X. Zheng, H. Schwetman, J. Lexau, P. Koka, and et al., "Computer systems based on silicon photonic interconnects,” Proc. IEEE, vol. 97, no. 7, pp. 1337-1361, Jul. 2009.

[2] D. Miller, "Device requirements for optical interconnects to silicon chips,” Proc. IEEE, vol. 97, no. 7, pp. 1166-1185, Jul. 2009.

[3] J. Van Campenhout, M. Pantouvaki, P. Verheyen, H. Yu, P. De Heyn, G. Lepage, and et al., " Silicon-photonics devices for low-power, high-bandwidth optical I/O,” in Proc. Integrated Photonics Research, Silicon and Nano-Photonics (IPRSN), Colorado, Jun. 2012, Paper ITu2B.

[4] G. Li, X. Zheng, J. Yao, H. Thacker, I. Shubin, Y. Luo, and et al., "25Gb/s $1 \mathrm{~V}$-driving CMOS ring modulator with integrated thermal tuning," Opt. Express, vol. 19, no. 21, pp. 20435-20443, Oct. 2011.

[5] P. Dong, R. Shafiiha, S. Liao, H. Liang, N. Feng, D. Feng, and et al., "Wavelength-tunable silicon microring modulator," Opt. Express, vol. 18, no. 11, pp. 10941-10946, May 2010.

[6] B. Guha, K. Preston, and M. Lipson, "Athermal silicon microring electro-optic modulator,” Opt. Lett., vol. 37, no. 12, pp. 2253-2255, Jun. 2012.

[7] P. Dong, S. Liao, H. Liang, W. Qian, X. Wang, R. Shafiiha, and et al., "High-speed and compact silicon modulator based on a racetrack resonator with a $1 \mathrm{~V}$ drive voltage," Opt. Lett., vol. 35, no. 19, pp. 3246-3248, Oct. 2010.

[8] W. Bogaerts, S. K. Selvaraja, P. Dumon, J. Brouckaert, K. D. Vos, D. Van Thourhout, and R. Baets, "Silicon-on-insulator spectral filters fabricated with CMOS technology,” IEEE J. Sel. Topics Quantum Electron., vol. 16, no. 1, pp. 33-44, Jan./Feb. 2010.

[9] X. Zheng, Y. Luo, J. Lexau, F. Liu, G. Li, H. D. Thacker, and et al., “2-pJ/bit (on-chip) 10-Gb/s digital CMOS silicon photonic link," IEEE Photon. Technol. Lett., vol. 24, no. 14, pp. 1260-1262, Jul. 2012.

[10] M. Pantouvaki, H. Yu, P. Verheyen, G. Lepage, W. Bogaerts, M. Moelants, and et al., "Lateral versus interdigitated diode design for 10 Gb/s low-voltage low-loss silicon ring modulators," in Proc. IEEE Conference on Optical Interconncects, Santa Fe, May. 2012, pp. 44-45.

[11] M. R. Watts, W. A. Zortman, D. C. Trotter, R. W. Young, and A.L. Lentine, "Vertical junction silicon microdisk modulators and switches," Opt. Express, vol. 19, no. 22, pp. 21989-22003, Oct. 2011.

[12] H. Yu, M. Pantouvaki, J. Van Campenhout, D. Korn, K. Komorowska, P. Dumon, and et al., "Performance tradeoff between lateral and interdigitated doping patterns for high speed carrier-depletion based silicon modulators,” Opt. Express, vol. 20, no. 12, pp. 12926-12938, Jun. 2012.

[13] X. Xiao, H. Xu, X. Li, Y. Hu, K. Xiong, Z. Li, and et al., "25 Gbit/s silicon microring modulator based on misalignment-tolerant interleaved PN junctions," Opt. Express, vol. 20, no. 3, pp. 2507-2515, Jan. 2012.

[14] P. Dong, W. Qian, H. Liang, R. Shafiiha, D. Feng, G. Li, and et al., "Thermally tunable silicon racetrack resonators with ultralow tuning power,” Opt. Express, vol. 18, no. 19, pp. 20298-20304, Sep. 2010. 